\title{
New CCTV approaches for environmental enforcement in Ireland
}

\author{
A. Palomo, A. McDermott, J. Dooley \& R. Farrell \\ Callan Institute, Electronic Engineering Department, \\ National University of Ireland Maynooth, Ireland
}

\begin{abstract}
According to a survey carried out in Ireland among the environment departments of all Local Authorities, closed-circuit television (CCTV) systems are the most widely used technology for monitoring and prosecuting illegal environmental activities. Despite the fact that new technologies have been proposed in recent years, CCTV remains the preferred option. However, commercial CCTV systems are not specifically designed to tackle environmental enforcement issues. From the survey analysis the main limitations of commercial CCTV equipment deployed to date for environmental enforcement are: night time monitoring, monitoring of high risk or hostile areas, and battery powered CCTV systems for short term or remote deployments. The objective for the research was to identify solutions to the CCTV issues raised in the survey. It focused on the adaptation, installation and testing of commercial CCTV equipment for environmental enforcement scenarios. For each scenario several technologies with different configurations were evaluated. Equipment employed in this work included: digital video cameras, infra red illuminators, analogue cameras with long range optical zoom and low power consumption cameras. The effectiveness of each solution was assessed on its ability to detect and record with accuracy individuals, or vehicle licence plates, and the offences committed in a cost effective manner. In conclusion, this research provided solutions for the three CCTV challenges raised in the survey by Irish Local Authorities. Consequently, new approaches to environmental monitoring are available to Local Authorities for future environmental enforcement in Ireland.
\end{abstract}

Keywords: CCTV, environment monitoring, prosecution. 


\section{Introduction}

In Ireland, the Environmental Protection Agency (EPA) and the Environment Departments of the Local Authorities are committed to the reduction of environmental pollution in all its shapes and forms [1]. Following the European Union recommendations on environmental enforcement, the Irish environmental legislation promotes the 'polluter pays' principle by which the activities or persons that cause environmental damage are held financially accountable for their actions [2-4].

The first step for applying the polluter pays principle is the identification of the activity and/or persons responsible for this illegal activity. The identification method varies depending on the activity and in most cases it involves the application of technology. For example, specialised sensors are used for the detection of Nitrates in water contaminated by agricultural sources, or air pollution produced by industry [1]. In these cases, the technology is used as a control mechanism rather than a polluter identification method since the responsible activities or persons are already known.

However, there are other environmental enforcement activities where the identification of the polluter is required in order to prosecute and obtain legal evidence of the offence. Illegal disposal of waste is one of these activities. It occurs in areas out of public view where the waste is dumped in an unauthorised way. This repeated behaviour in a specific area leads to what is known as an illegal dumping 'black spot'. In these cases, visual evidence of the polluter and the illegal action provided by technology, such as close-circuit television (CCTV) systems, or a member of the public is necessary to carry out a legal prosecution.

\section{Environmental enforcement issues in Ireland}

The environmental enforcement activities carried out by Irish Local Authorities and the Irish EPA can be mainly classified in four categories: waste enforcement, water enforcement, air and noise enforcement and large industrial activities [1]. Each Local Authority is responsible for assessing which of these areas represent the higher risk in their jurisdiction and dedicate the necessary resources for their monitoring and enforcement.

Despite the different geographical nature of the Local Authorities, waste represents the greatest challenge among all enforcement activities for each of them. According to a survey study carried out nationwide with the environment departments of all the Local Authorities, the top ten environmental enforcement issues faced are directly linked to waste activities [5]. Out of the 34 Local Authorities in Ireland, 17 of them (50\%) participated in this study. Figure 1 shows the results collected from the surveyed Local Authorities when they were requested to name their most significant five environmental enforcement issues.

Among all the issues shown in Figure 1, illegal waste disposal (also known as fly tipping) clearly stands as the main issue. According to [6], fly tipping can be described as the indiscriminate dumping of bags containing household waste, 


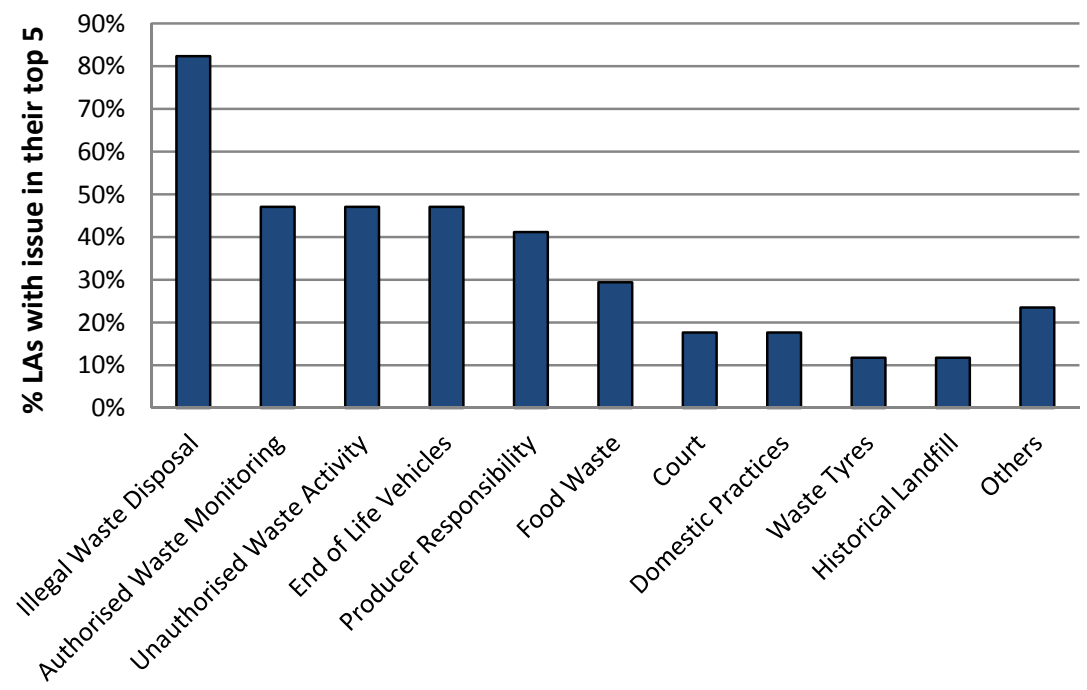

Figure 1: $\quad$ Irish Local Authorities Main Environmental Issues [5].

waste electronic and electrical equipment, end-of-life vehicles (EOL), pallets, and construction and demolition (C\&D) materials and other waste streams. Fly tipping has a random nature since any location can be targeted for the disposal of waste. However, in the rural areas of Ireland fly tipping tends to occur in remote areas with difficult access like bogs, forestry areas and commonage land. For urban areas, fly tipping cases mainly takes place in recycling centres, normally designated for bottles and clothes collection, and civic amenity areas.

Specifically for the household waste case, the number of illegal dumping cases has increased due to socio-economical factors such as the introduction of a 'pay-as-you-throw' rubbish collection system [7, 8]. This system aims to increase the involvement of house holders in reducing the amount of rubbish they produce by dictating their rubbish collection fees accordingly. However, this scheme can lead to illegal waste disposal by the house holder, either themselves or through illegal waste collectors.

In Ireland, the environment departments of the 34 Local Authorities are the first point of contact for environmental complaints. It is estimated that the Irish Local Authorities receive 70,000 complaints every year in relation to a broad range of environmental issues [1]. Local Authorities and the EPA have dedicated call lines for receiving information from the public about environmental issues. For the particular case of the national call line known as 'Dump the Dumpers', $75 \%$ of the calls received are related to fly tipping and burning of waste [1].

The problem with illegal waste disposal is not unique to Ireland, but a generalised issue which occurs in other countries such as the UK. In the UK, for example, illegal waste disposal tops the rank of environmental offences with $61 \%$ of overall offences [9]. 


\section{Environmental enforcement technologies used by Irish Local Authorities}

The Irish legislation on environmental enforcement, with the Waste Management Act and the Litter Management Act as its main documents [2, 3], aligns with the European environmental law in terms of the 'polluter pays' principle [4]. This means that the activities or persons that cause environmental damage are held financially accountable for their actions. For example, most part of the illegal household waste disposal would be prosecuted under the Litter Management Act which establishes a maximum fine of up to €3,000 for the offenders [3].

A crucial part of the offender prosecution process is the identification of the responsible persons committing the illegal activity. This is where technology plays a crucial role in the field of environmental enforcement. In the same survey study cited in Section 2 [5], the Irish Local Authorities were asked to name the technologies that they use, or have used, to tackle illegal waste activities. In addition, they were requested to distinguish if these technologies were used in rural or urban areas. The results collected from the surveyed Local Authorities are shown in Figure 2.

From the results it can be observed how CCTV systems are the most widely used technology in both urban and rural areas with $100 \%$ of the Local Authorities either using this method permanently or temporally in specific areas associated with illegal dumping. Other technologies also employed, but in much less proportion, are satellite imaging (normally low resolution images obtained from systems like Google Earth), sensors for detecting factors such as contaminant substances in the water of rivers and lakes, and aerial photography

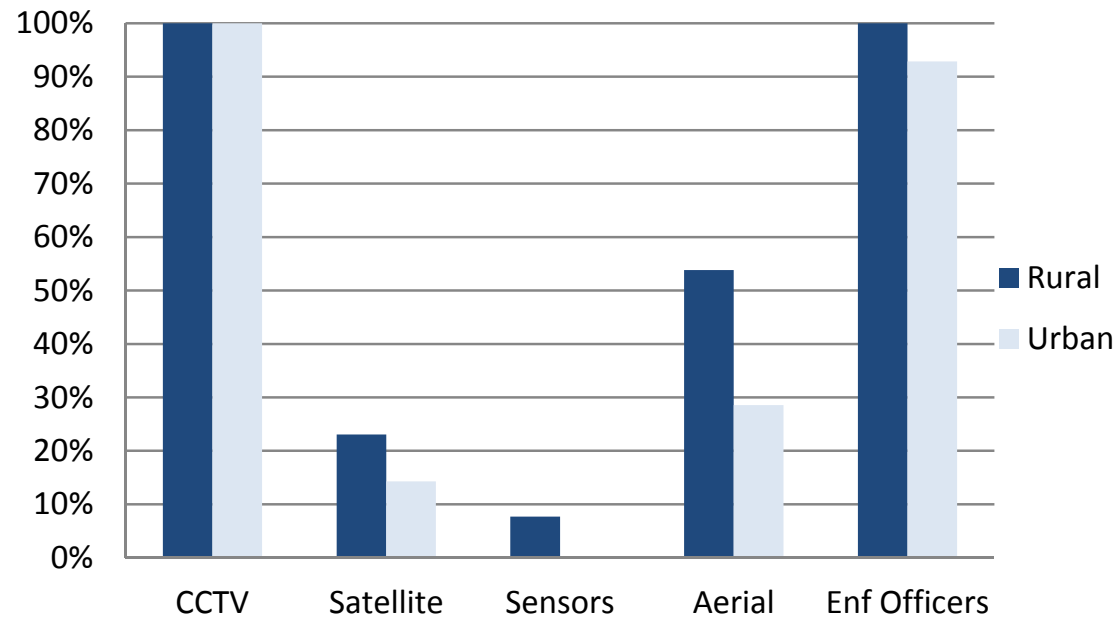

Figure 2: $\quad$ Environmental enforcement technologies used by Irish Local Authorities [5]. 
taken from helicopters or unmanned aerial vehicles (UAV) such as quadcopters [10]. In addition to these technologies, the surveillance carried out by enforcement officers was also included in this comparison due to its importance in all surveyed Local Authorities.

The main factors which make CCTV the preferred technology method are: its reduced price in comparison with the sensors or aerial photography services, the immediate and updated availability of the images in comparison with outdated systems like historical satellite images, or its remote monitoring using internet or wireless connections which allows the enforcement officer to remotely monitor the surveyed area. In addition, CCTV systems are the only solution which allows recording of the illegal action while it takes place and not the consequences, a point that is crucial in the prosecution process. For example, under the Litter Management Act, if the licence registration plate of a vehicle used for an illegal environmental activity is recorded, the owner of the vehicle can be prosecuted as a responsible person [3].

\section{Environmental enforcement CCTV challenges}

Despite the wide use of CCTV systems by the Irish Local Authorities in the field of environmental enforcement, the performance of these systems can be less than desirable in some scenarios. One of the reasons for this performance is due to the fact that many of the Local Authorities employ commercial CCTV systems which are not designed for environmental monitoring.

Three main challenging scenarios regarding CCTV were expressed by the Irish Local Authorities in the survey study [5]. First, the night time monitoring of black spots represents a challenge for CCTV systems. In cases where offenders use vehicles to transport waste, efficient CCTV systems which allow identifying both the illegal activity and the licence plate registration of the vehicle are required. For this particular case the two main challenges are how to overcome the low light conditions of the scene and how to avoid the vehicle headlights saturating the image sensor of the camera and preventing licence plate recognition.

Second, the monitoring of high risk or hostile areas creates a challenge for CCTV systems. Typically these are populated areas where the monitoring equipment has a high probability of being vandalised or stolen.

Finally, the third scenario focuses on temporal or remote CCTV deployments which can not avail of mains power, and consequently they need to be powered by batteries. In general, CCTV systems formed by cameras and recording devices consume a relatively large amount of power and can only work for a few hours on battery power. Therefore, they do not represent a viable option when mains power is not available.

Despite the difficulty of the three scenarios for commercial CCTV systems, the appropriate combination of specific monitoring devices could lead to successful results. Consequently, a research study to investigate the optimum combination of CCTV devices to overcome the difficulties of these scenarios was completed. The performance of the devices was measured in terms of 
licence plates and activity recognition. An additional criterion was to minimise the overall cost of the CCTV system deployed.

\subsection{Night time monitoring}

Night time CCTV solutions imply a much higher challenge than day time systems since the low light conditions and the front headlights of the vehicles make it difficult to identify both the illegal activity and licence plate. In this respect, two technology solutions were deployed and tested for night time monitoring.

The first solution explored was an analogue system consisting of a 600 TV lines (TVL) resolution high end camera with infrared lights, a licence plate recognition (LPR) camera and a digital video recorder (DVR). The 600 TVL camera had the mission of capturing the illegal actions both during the day and at night, and assisting the LPR camera in capturing licence plates during the day time. In addition, this camera had the capabilities of blocking bright sources of light, such as vehicle headlights at night or sun reflections in day time, to provide a clear video image. On the other hand, the LPR camera was fully dedicated to the task of identifying licence plates in both day and night conditions. The DVR was purely used for the recordings and playbacks of the captured images.

The second solution was a digital system consisting of a 3.1 megapixel (MP) camera with infrared lights, an external infrared illuminator and a network digital video recorder (NVR). In this system the camera was had to capture both activities and licence plates, while the external illuminator would enhance the light in the scene at night time. In addition, it would reduce the effect of bright light sources on the camera sensor such as vehicle headlights.

The analogue CCTV solution was deployed in a controlled environment where vehicles and persons could be monitored. The cameras where situated approximately 15 meters away from the main point of interest in the scene. At night, the scene was partially illuminated with street lights. Figure 3 shows some of the images obtained from both cameras during the day and at night. In all the images the licence plates were partially obscured for confidentiality.

The 600 TVL camera provided colour images both during the day and at night (Figure 3(a)) and Figure 3(c)). The bright light blocking function of the camera was effective at blocking strong sources of light and enabling the entire scene to be observed. In Figure 3(a)) the 600 TVL camera blocks the sun reflection on the car while in Figure 3(c)) the camera blocks the car headlights in a night scene. However, the camera did not allow licence plate recognition during day or night.

The LPR camera provided black and white images during the day and at night (Figure 3(b)) and (d)). From Figure 3(b)), the LPR camera was able to read licence plates from a distance of 15 meters during the day. The licence plate could be recognised when the car was at an angle to the camera. At night time, as seen in Figure 3(d)), the licence plate recognition distance was reduced to 10-12 meters. In this case, it was necessary that the car completely faced the camera. The licence plate could be read even if the car has the headlights on. The overall 


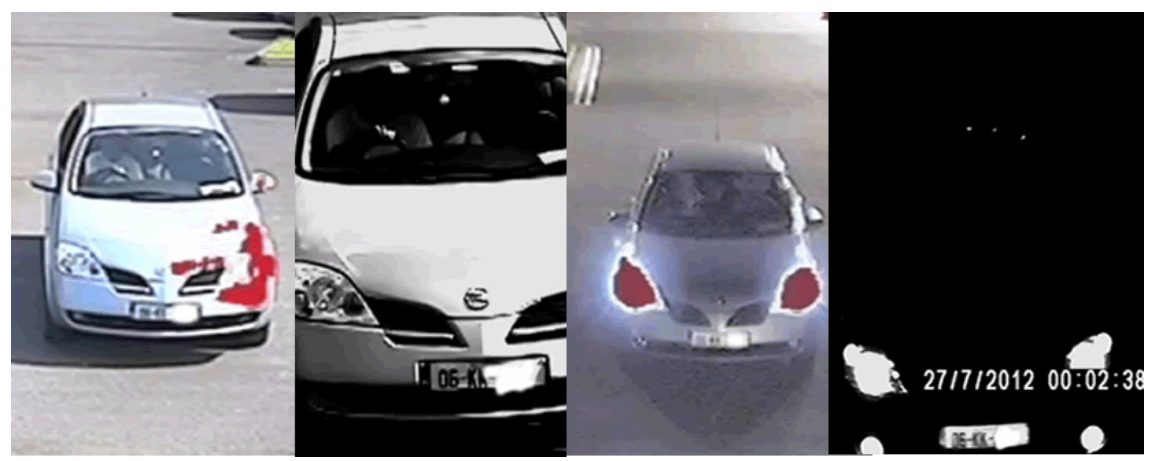

Figure 3: $\quad$ Examples of 600 TVL and LPR cameras images. From left to right: (a) 600 TVL camera in daylight, (b) LPR camera in daylight, (c) 600 TVL camera at night time, d) LPR camera at night time.

action in the scene could only be observed during day light. At night time only the car plate could be seen whereas the rest of the scene was completely dark.

Unlike the analogue CCTV solution, the digital CCTV set was tested in complete darkness. In the shorter distances, between 10 and 20 meters, the camera and the illuminator were able to display correctly the licence plate of the vehicle even though the headlights were on (Figure 4(a)). The actions of people passing by the scene could also be observed (Figure 4(b)). When the camera was working alone with no external illuminator, it could display the plates and the action in darkness, but not the plates with the headlights of the car on.

The settings for camera exposure and image brightness were adjusted so that the combination of the MP camera and the illuminator provided successful licence plate recognition for distances up to 33 meters in complete darkness (Figure 4(c) and Figure 4(d)). Without the assistance of the external illuminator, the digital camera could recognise licence plates over the same long distance but with poorer image quality.

In conclusion, the performance of the analogue solution system was worse than initially expected. The LPR camera capture licence plates numbers both at day and night time. However, the licence recognition range was smaller than initially expected, especially at night time. The 600 TVL camera had a good performance for monitoring activity, providing colour images both day and night, but could not recognise licence plates even at short ranges (10-12 meters).

On the other hand, the digital solution provided an effective performance both day and night allowing licence plate and activity recognition over distances up to 33 meters at night time. The external illuminator was shown particularly effective when monitoring scenes with vehicle headlights. From an economic perspective, the digital solution is approximately $30 \%$ more expensive than the analogue solution, but it is more effective and reliable. 

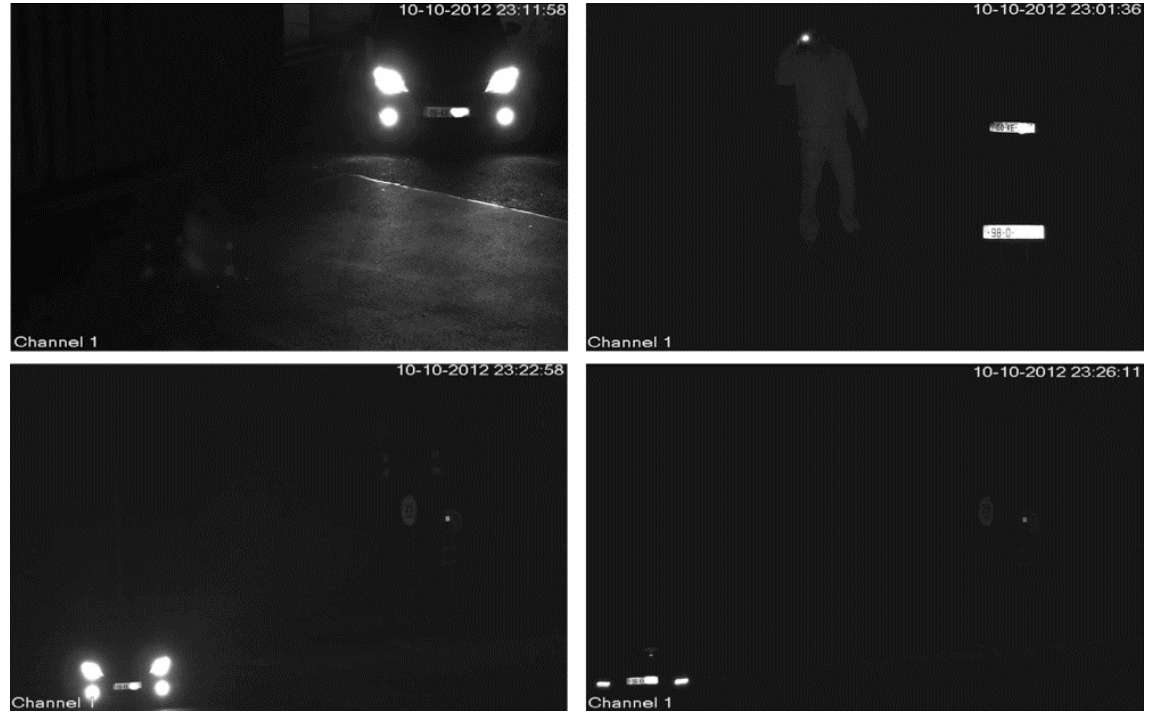

Figure 4: $\quad$ Examples of digital CCTV system images, from left to right and top to bottom: (a) camera + external illuminator licence plate recognition with the car situated $20 \mathrm{~m}$ away and headlights on (b) camera + external illuminator licence plate recognition and action recognition in complete darkness; (c) camera + illuminator licence plate recognition car 33m away and headlights on (d) only camera licence plate recognition 33m away and back lights on.

\subsection{Monitoring of high risk or hostile areas}

For the purpose of this paper, hostile areas refer to areas where CCTV equipment is at a very high risk of being stolen or damaged. Current practices include mounting CCTV cameras in the hostile area on high poles and housed in protective cages.

An alternative solution which was investigated was to place the CCTV equipment in a safe area away from the hostile environment. The idea was to set up the equipment and monitor from a safe distance so that occupants of the hostile area were not aware of the camera location. In order for this to be successful, two criteria needed to be satisfied: a long range camera with high optical zoom and direct line of sight to the hostile area.

Field trials were completed using an analogue $37 \mathrm{X}$ optical zoom camera and a DVR for recording footage. The area of interest was 150 to 180 meters away from the camera. Images in Figure 5 show the capability of the camera to recognise licence plates up to a distance of 180 meters away (Figure 5(c)). One observation with long range CCTV equipment is that the camera must have a very stable mounting. Otherwise, windy conditions result in camera vibration 
which is compounded when the camera is set at high zoom configurations, resulting in unsuccessful licence plate recognition.

An additional function of the camera was its ability to monitor a wide view and once movement occurred, it could be programmed to zoom into that area and remain there so long as movement continued. For example, Figure 5(a) shows a wide scene monitored with the camera using a $1 \mathrm{X}$ optical zoom. When movement was detected in the scene, the camera zoomed to area under interest with a previously programmed 25X zoom, as shown in Figure 5(b).

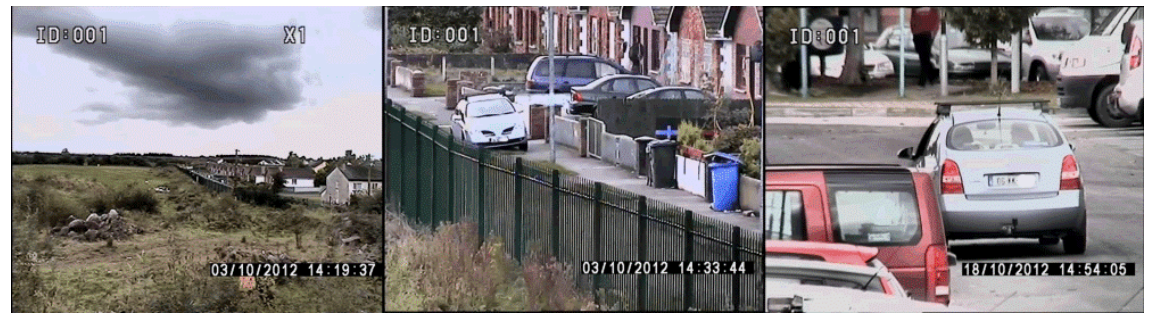

Figure 5: Long range 37X optical zoom camera images: (a) field of view with no zoom, (b) $25 \mathrm{X}$ zoom into area of interest, (c) $37 \mathrm{X}$ zoom with successful licence plate recognition over 180 meters.

In conclusion, analogue cameras with powerful optical zooms offer an effective solution for monitoring high risk or hostile areas since the equipment can be safely deployed outside this area.

\subsection{Battery powered CCTV for short term or remote deployments}

Illegal waste disposal and fly tipping of rubbish often occurs in remote areas where there is either no mains power supply available for CCTV equipment or the cost of connecting to a mains supply is significant and also attracts undesired attention. This is a major concern for local authorities in Ireland and beyond.
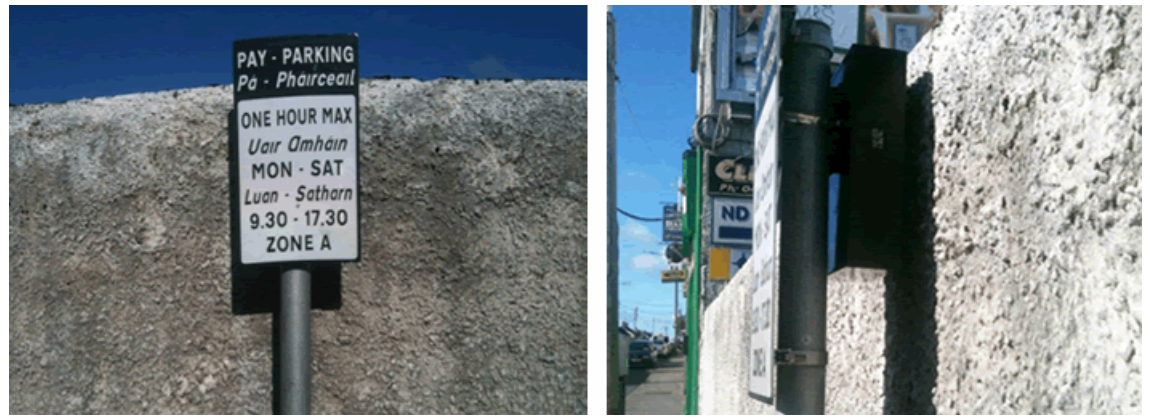

Figure 6: Covert low power CCTV system mounted behind street sign. 
As a consequence, these areas tend not to be monitored at all or else monitored for very short time periods using CCTV equipment powered by car/truck batteries. By its nature, commercial CCTV equipment consumes large amounts of power and therefore a regular $12 \mathrm{~V} 40 \mathrm{AH}$ car battery will last less than 24 hours in the field. So, although it works, the solution is not very satisfactory.

\subsubsection{Low power CCTV system}

As an alternative to regular CCTV powered by a car battery, this research focused on identifying very low power consumption devices. A mini 600TVL analogue camera coupled with a mini DVR was chosen, with the entire configuration only consuming 250mA when operational. This set-up was powered using two $12 \mathrm{~V}$ DC $7 \mathrm{AH}$ batteries in parallel and all the equipment was housed in a box with a relatively small form factor (300mm x $200 \mathrm{~mm} \times 70 \mathrm{~mm}$ ).

Based on its size, this set-up enabled easy installation as a covert CCTV system and its configuration enabled it to operate for 50 hours on the small battery pack. Figure 6 shows the solution mounted behind a small street sign and not perceived as a regular CCTV system.

Although this solution achieved a small form factor and long battery life, the image quality provided by the mini 600 TVL was worse than a standard 600 TVL camera like the one presented in Section 4.1. Therefore, it was found only suitable for daylight applications in a short range.

\subsubsection{Trail camera system}

Four high end cameras were tested during this research for remote deployments. They all shared common features such as: triggered by a passive infrared (PIR) sensor, programmable number of pictures per trigger, high image resolution between 3 and $12 \mathrm{MP}$ and battery life allowing between 20,000 and 40,000 images.

Some systems offered additional features such as image transmission via email or multimedia message (MMS) while others had greater programmability options. Their ease of installation coupled with the high quality images enables them to be a very practical solution for areas of up to 400 triggers per day, such as recycling centres, derelict buildings or remote areas.
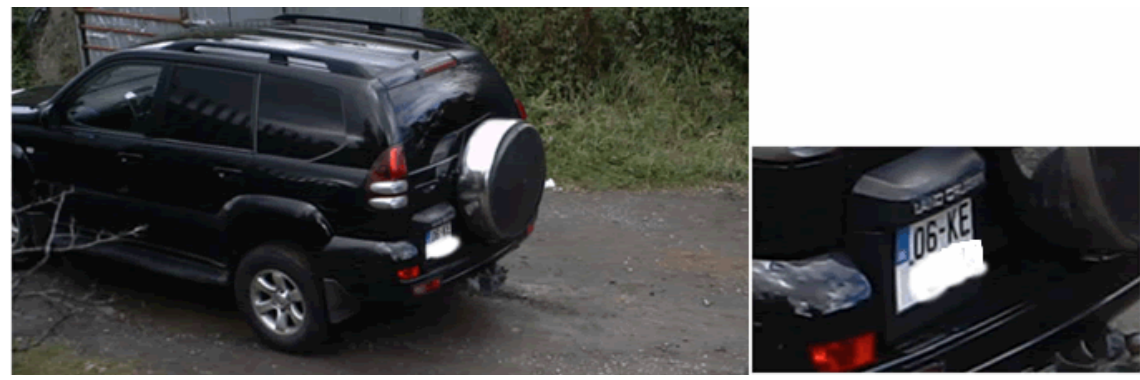

Figure 7: $\quad$ Licence plate recognition at 20 meters using trail camera. 
Daylight monitoring was found effective, while night time only provided a good performance in areas where background lighting, such as street lights, was present for assisting the infrared illuminators on the trail cameras.

The field testing showed the trail camera PIR sensors were activated with movement up to a distance between 20 and 25 meters. The detection zone did not extend reliably beyond $25 \mathrm{~m}$. These applications required that vehicle licence plates could be recognised in the images taken. From the tests, it was found that a camera set to 3MP resolution could read licence plates up to 30 meters away. The increase in the pictures resolution from 3MP to 5MP or 12MP did not noticeably extend the distance that plates could be recognised. As an example, Figure 7 shows a vehicle 20 meters away from the camera which licence plate was clear to read when the image was magnified.

An alternative to the trail camera is the time lapse camera. This camera captures an image at a programmed frequency regardless of activity, not being triggered by a PIR sensor. Time lapse cameras offer advantages versus trail cameras in applications where images of a scene are required at a specified interval regardless of activity. In addition, because they do not employ PIR sensors, they are easier to camouflage. In particular, the model tested was able to take one million images on a set of batteries (8xAA batteries). It was used to detect incidents of illegal dumping at a litter black spot on an urban pathway where footfall was high and it proved very effective in this application. Some limitations with this model were the lower image resolution (1MP) and the lack of infrared lights for night time monitoring.

\section{Conclusions}

CCTV has been unanimously adopted by Local Authorities across Ireland to support their environmental enforcement efforts. However, this technology is less than effective for many scenarios they experience. A fundamental challenge with the technology is the fact that commercial CCTV designed for security applications is expected to perform for environmental enforcement monitoring even though requirements are very different.

This paper clearly demonstrates that the main CCTV challenges experienced by Local Authorities in Ireland can be overcome by careful and appropriate combination of products tailored for specific scenarios. For example, night time monitoring with licence plate identification can be carried out in almost total darkness conditions with the combination of a MP digital camera and an external infrared illuminator. In addition, for the monitoring of hostile areas, long distance CCTV surveillance was proven successful in terms of image quality when commercial CCTV cameras with large zoom capabilities were employed. Finally, the problem with low duration battery powered CCTV systems was solved with the application of a very low power consumption camera and DVR combination, or the use of standalone trail cameras. 


\section{Acknowledgements}

The authors would like to thank the EPA for their generous support for the research presented in this article through the Strive program and the research grant 2011-ET-MS-11. They would also like to thank all Irish Local Authorities for their collaboration, in particular Cavan, Fingal and Westmeath.

\section{References}

[1] EPA, Focus on Environmental Enforcement in Ireland - A Report for the Years 2006-2008. 978-1-84095-323-7, 2009.

[2] Department of Environment, Community and Local Government, Waste Management Act. No. 10/1996, 1996.

[3] Department of Environment, Community and Local Government, Litter Pollution Act. No. 12/1997, 1997.

[4] European Commission, Directive 2004/35/CE of the European Parliament and of the Council on Environmental Liability with Regard to the Prevention and Remedying of Environmental Damage. EC 2004/35/CE, 2004.

[5] A. McDermott, et al., National Study on Environmental Waste Enforcement: Issues, Best Practices and Technologies. Environ 2013, Galway, Ireland, 2013.

[6] EPA, The Nature and Extend of Unauthorised Waste Activity in Ireland. 184095-170-2, 2005.

[7] L. Dunne, et al., An investigation into waste charges in Ireland, with emphasis on public acceptability. Waste Management, vol. 28, pp. 28262834, 2008.

[8] EPA, A Nationwide Review of Pay-By-Use Domestic Waste Collection Charges (PBU) in Ireland: Extensive Survey Findings. 1-84095-235-0, 2007.

[9] D. Stott, Environmental Enforcement in the UK. Journal of Environmental Monitoring, vol. 11, pp. 470-474, 2009.

[10] M. Lega, et al., Illegal Dumping Investigation: A New Challenge for Forensic Environmental Engineering. WIT Transactions on Ecology and The Environment, vol. 163, 2012. 